\title{
Research on Algorithms of Spatial-Temporal Multi-Channel Allocation Based on the Greedy Algorithm for Wireless Mesh Network
}

\author{
Yiyong Wang $^{{ }^{*}}$ and David Baker ${ }^{2}$ \\ ${ }^{1}$ ZheJiang Technical Institute of Economics, Digital Information Technology Institute, Hangzhou 310018, China \\ ${ }^{2}$ Indiana University - Purdue University Fort Wayne, United States
}

\begin{abstract}
With the development of mobile Internet and wireless network as well as the rapid increasing number of users, people's demand for broadband is increasing sharply, making further capacity expansion of the network the major problem for researchers. Wireless Mesh network has the advantages of high network coverage and flexible networking, especially multi-hop wireless network, its important networking format takes full use of the feature of orthogonal channel to raise the throughput of wireless network and utilization efficiency of broadband dramatically and make the channel resources allocation more flexible. This paper points out the advantages and disadvantages of Mesh network by analyzing some relatively classic algorithms based on the research of Mesh network technology in order to increase utilization efficiency of orthogonal channel further. At the same time, it proposes Spatial-Temporal Multi-Channel Allocation Based on the Greedy Algorithm for Wireless Mesh Network which takes the channel allocated in previous slot time into consideration and allocates channels to different ports to reduce the switching time and other costs. Giving the direction of nodes is up stream, it requires well-functioning nodes near the gateway. Any network fault will influence other nodes near these gateways. This allocation realizes the communication between nodes on original channel nodes, increasing utilization efficiency significantly and reducing the burden of parent node. In the end, this paper makes a conclusion of the main work.
\end{abstract}

Keywords: Greedy algorithm, spatial-temporal channel allocation, wireless mesh network, multi-channel allocation.

\section{INTRODUCTION}

With the rapid development of wireless communication and $4 \mathrm{G}$ technology, traditional wired LAN cannot satisfy the increasing need of times, more people pay their attention to wireless network, and considering the huge market demand for wireless network, many developers step into research field of wireless Mesh. Meanwhile, higher hardware technology and lower price provide support for building a wireless city. A survey shows that the number of wireless network in the world rose by leaps and bounds in just a few years. Fig. (1) shows the global deployment diagram of Mesh network distribution, in which most of them are in the developed countries. But because of the development of Asian technology and people's increasing demand of broadband, Asian proportion in this diagram is getting lager and lager.

With the popularity and application of wireless Mesh network, the number of tasks bore by wireless Mesh is increasing too. But giving the randomicity of node's movements and the isomerism of client in backbone network, to find a high-efficiency channel allocation format to guarantee that nodes connections, terminal connections, geographical

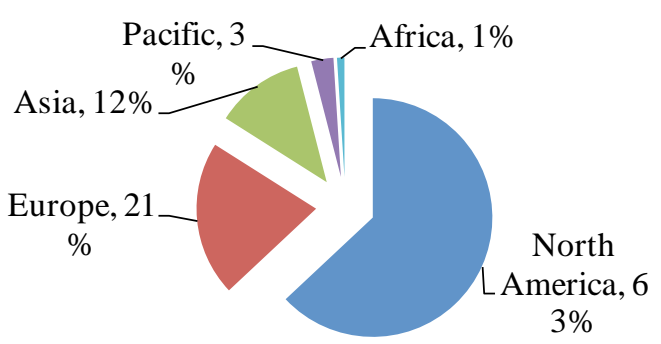

Fig. (1). Global deployment diagram of Mesh network distribution.

position connections and non-direct nodes are connected and receive data flow so as to improve the performance, huge capacity and robustness of the whole network is a hot field for wireless Mesh network research. This paper proposes Spatial-Temporal Multi-Channel Allocation Based on the Greedy Algorithm on the basis of analysis of wireless Mesh technology, and introduces multi-channel allocation technology to deepen people's understanding of multi-channel wireless technology, and provides better solution according to the important principle influencing nodes allocation of wireless channel.

The rapid development of wireless Mesh network gets the whole city covered, accelerates the development of traffic guidance in city, enhances abilities related to urban informatization, and provides better service for netizen. 


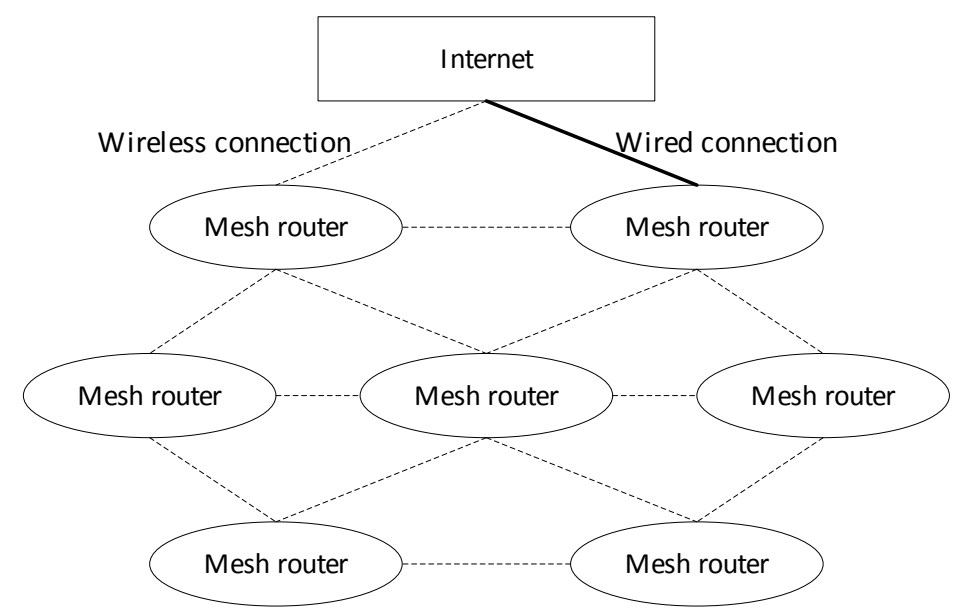

Fig. (2). Diagram of backbone network main structure.

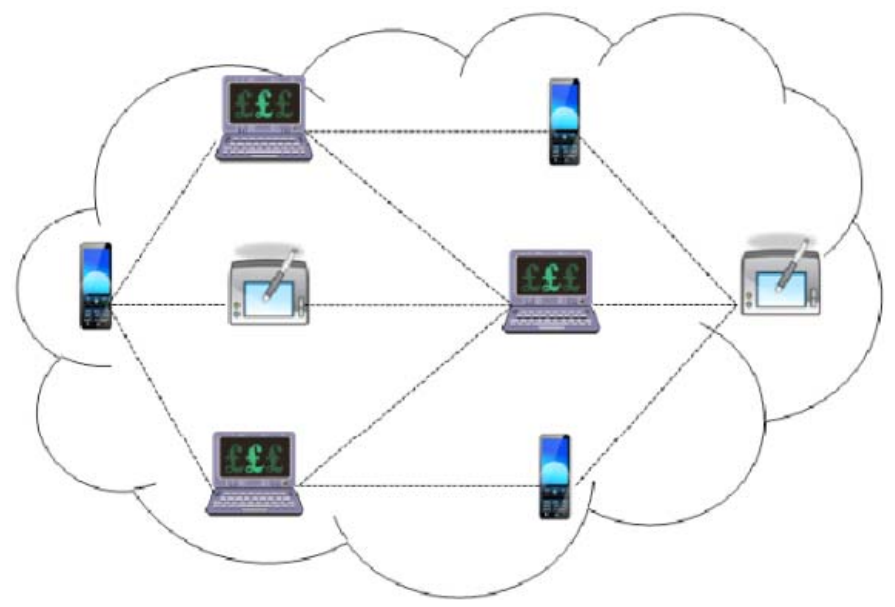

Fig. (3). Client Mesh network structure.

\section{RESEARCH ON WIRELESS MESH TECHNOLOGY AND MULTI-CHANNEL ALLOCATION}

Wireless Mesh networks (WMN), as a kind of wireless network, is different from wireless LAN and Ad Hoc network, a multi-top network with self-organization function. Wireless Mesh network firstly used in American military is gradually applied in people's daily lives and production mainly thanks to the support of internet companies which contribute a lot to the research and development of it.

\subsection{Wireless Mesh Technology}

Wireless Mesh network [1] consists of Mesh nodes with route function and information transmitting nodes rather than client nodes without route function. Wireless Mesh networks can be classified into backbone network, client wireless Mesh network and mixing network according to the function of nodes. All nodes in backbone network have route function and equal positions and functions, can connect to clients and receive, transmit and send data. This network stores a lot of information from clients, and the capacity of storage affects channel allocation. Its main structure is as shown in Fig. (2).

All clients in client Mesh network are equal, too, making it a mobile Ad Hoc network which can realize router alloca- tion independently. Giving there is no routers between client servers nodes, client servers cannot be connected to Internet directly by net inside client, but can be connected through transmit of other clients. Client Mesh network structure is as shown in Fig. (3).

Mixing wireless Mesh network mixes the above two networks, every node in this network can be connected to the net, even without backbone network. Backbone network in this network also provides connectedness between clients, such as sensor network, Internet and WIMAX, improving coverage rate and connectedness of the whole wireless Mesh network. It's structure is as shown in Fig. (4).

Wireless Mesh network has the advantages of WLAN and Ad Hoc network, can cover a larger range with limited transmitting power, disposing the congestion problem. Mesh network and WLAN need the support of infrastructure, while Ad Hoc doesn't; Ad Hoc network and Mesh network can realize direct communication between nodes, while WLAN cannot; Ad Hoc network and Mesh network can realize multi-hops and self-organizations, while WLAN cannot. Wireless Mesh network has many advantages, including easy and convenient deployment and settlement, the ability to realize 


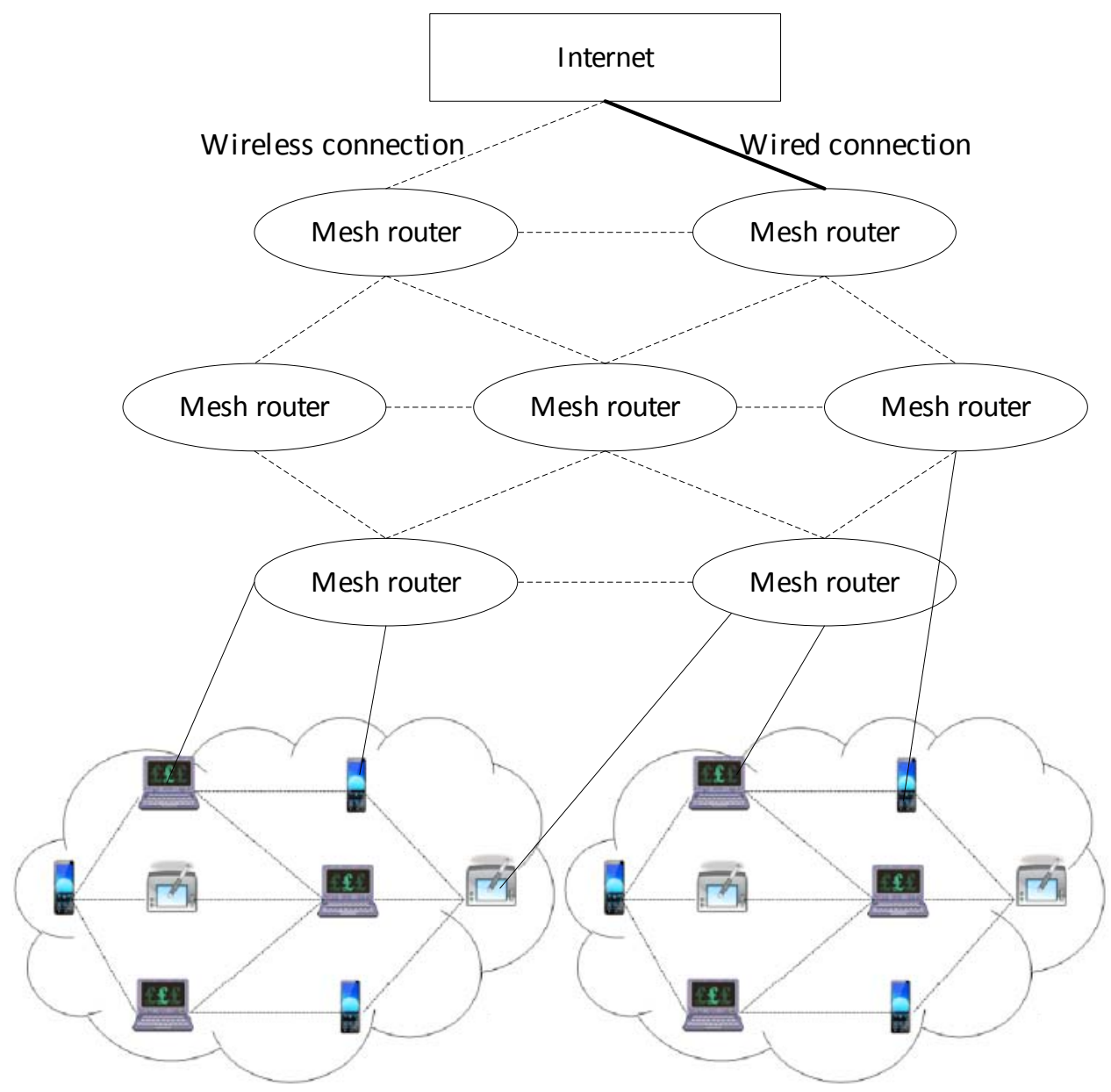

Fig. (4). Structure of mixing wireless Mesh network.

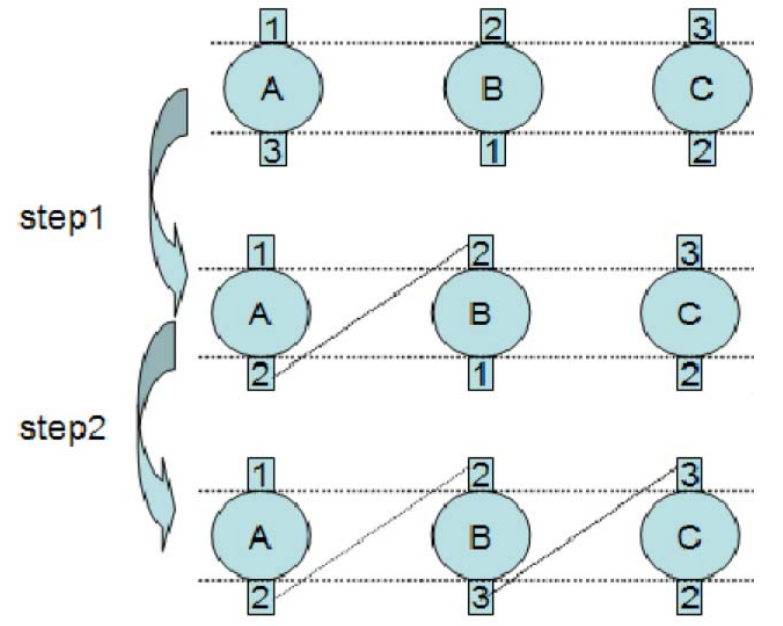

Fig. (5). Diagram of channel allocation algorithm.

invisual distance communication, strong robustness, better extendibility and good network compatibility, usable broadband. Nevertheless, its device compatibility is not that good, as a result its delay is large and security is low. So this network is mainly used in home, school, leisure places and temporary deployment.

\subsection{Multi-Channel Allocation}

All nodes are classified into two parts by mixing allocation channel [2], one part for keeping the allocation results indeformable, the other for connection required by dynamic network. As is shown in Fig. (5), node A needs to send tasks to node $\mathrm{C}$ via node $\mathrm{B}$, and channels of node $\mathrm{A}, \mathrm{B}, \mathrm{C}$ named $1,2,3$ respectively, are fixed and tunable. During the process, node A changes channel into Channel 2, and node B supervises the signal on Channel 2, then node A can send data to node B. Secondly, node B changes its channel into Channel 3 to transmits the data from node A to node $\mathrm{C}$.

Interface matching channel [3] is designed to ensure network's entire performance, increase entire throughput, reduce noise hindrance between nodes and short network delay. Giving that interfaces of wireless Mesh network under current situation cannot rise unlimitedly, the collateral mode of multi-channel is adopted to raise throughput of network nodes and keep the integrity of network.

Distributed channel allocation is suitable for gateway distribution [4], its allocation is to build topological structure at gateway node and allocate channels according to the volume of information acquired. The channel allocation is divided into two parts: interface binding and interface channel binding. 
Channel allocation should take self-utilization of the channel into consideration, and then exchange information with channel in the neighbor, at last, choose a better channel to transmit it. This algorithm uses directional antenna to increase entire throughput of the network significantly.

\section{RESEARCH ON SPATIAL-TEMPORAL MULTI- CHANNEL ALLOCATION BASED ON THE GREEDY ALGORITHM}

Interface channel allocation of wireless Mesh network can realize rational use of broadband only with the support of certain physical conditions. With the increasing network coverage range and nodes, linear programming used to tackle this problem may generate worse problem as the geometric growth, making multi-channel allocation into abstract image point restriction.

\subsection{Spatial-Temporal Multi-Channel Allocation}

Many factors should be considered in multi-channel allocation, for which researchers in the world have made a lot of researches and proposed many rational solutions. New allocation framework model downsizes and tackles the NP [6] problem by recursion, but there will be NP problem if network is too huge. Wireless Mesh network channel allocation tackles this problem spatiotemporally instead of spatially, that is allocating the next slot time to this node to bear the data from the previous node when the topological structure keeps stable in a certain time.

Route are chose by routers according to the number of node pairs [7] the network can bear. On the basis of the selected route, make a slot diagram of all nodes on first hop. Then test the previous slot, randomly delete the link whose variable larger than the radio frequency of this node, and put this link into the next slot for allocating. If there is link conflict in the test of the next slot, coloring graph can be used in channel allocation. If all the nodes in sub-slot are allocated into links and there are extra channel available, properly add the border of the second hop for channel allocation. After that, make a slot diagram of unallocated routers in the second hop and other link nodes, and repeat again and again until all of the all ocation are finished, as is shown in Fig. (6).

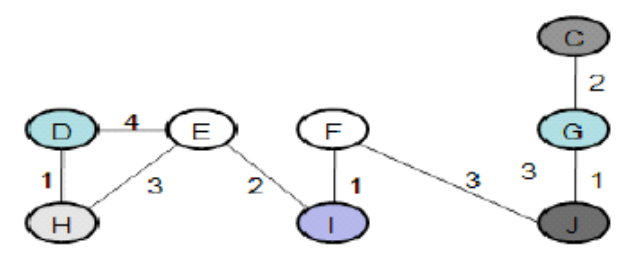

Channel allocation of the first sub-sequence chart

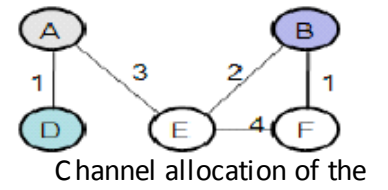

second sub-sequence chart

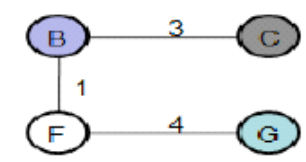

Channel allocation of the third sub-sequence chart
Fig. (6). Sub-slot allocation.
This algorithm has the following advantages [8]: (1) select communication route according to the router to simplify problem, making infeasible method which worsens the problem as the geometric growth feasible; (2) chancel some shared channels which are used to keep channel integrity, tackling "ripple" effect; (3) balanced load and flow in backbone network makes router's algorithm stable as well as nodes bearing tasks, making the use of channel allocated once longer.

\subsection{Spatial-Temporal Multi-Channel Allocation Based on the Greedy Algorithm}

The algorithm in this paper regards twice of the communication range as interference distance, allocates channel to each sub-slot node by linear allocation method, assuming all routers are distributed equably on the flat and all radio frequency interfaces on all nodes are usable. According to the principle of greedy algorithm [9], channels allocation can continue infinitely with channel working well.

Step 1: In abstract network connection diagram, determine communication nodes pair according to the volume of task and flow bore by the network and determine communication route, make Chart $\mathrm{G}$ of the first sub-slot with the first hop on router and the node initiating communication as starting point.

Step 2: Test Chart G to make sure all nodes conform the restriction of radio frequency interface. Delete node and its link, and put it into next slot if this node doesn't conform the restriction.

Step 3: Allocate the channels in step 2, assume this is the sub-chart of the first slot, adopt linear allocation method, randomly delete and put it into next slot if beyond the quantitative restriction.

Step 4: properly add the links of next hop into this chart. If there is still channel interference, allocate channels until all the sending and receiving nodes are allocated.

\section{CONCLUSION}

With the rapid development of wireless communication technology, the information age has come. Network changes people a lot, from production to study. People's dependency of wireless technology accelerates the development of it. Giving more and more people put their attention on wireless technology, wireless Mesh technology is created. Wireless Mesh technology has the advantages of traditional LAN and Ad Hoc network, and tackles the building problem of LAN, expanding the net coverage range. With the increasingly improved hardware technology and lower price of wireless technology, wireless Mesh is gradually used in many fields. Inevitably, some problems appear, for example, topological structure problem, so a rational channel allocation strategy is needed to improve net performance.

\section{CONFLICT OF INTEREST}

The authors confirm that this article content has no conflict of interest. 


\section{ACKNOWLEDGEMENTS}

Declared none.

\section{REFERENCES}

[1] X. F. Xiong, F. Yin, G. X. Yue, and J. S. Liu, "Research of spatialtemporal multi-channel allocation based on the greedy algorithm for wirless Mesh network", Telecommunications Science, vol. 11, pp. 98-102, 2012.

[2] G. F. Wu, Z. M. Ji, and J. Zhang, "Cognitive Wireless Mesh Networks", Journal of Information Engineering University, vol. 4, pp. 429-433, 2010.

[3] L. Ma, Research on Resource Allocation and Dispatching Algorithm of Wireless Mesh Network. Wu Han: Doctoral dissertation of Huazhong University of Science and Technology, 2010.

[4] Z. H. Ge, T. S. LI, and J. C. Zhang, "Research on channel assignment algorithm for wireless mesh networks", Journal of Guangxi
University (Natural Science Edition), vol. 35, no. 6, pp. 1002-1005, 2010.

[5] K. Ramachandran, E. Belding, and K. Almeroth, "Interference aware channel assignment in multi-radio wireless mesh networks", in Proceeding of IEEE INFOCOM, pp. 1-12, 2006.

[6] Q. Q. Wang, H. J. Li, X. G. He, and M. Xu, "Adaptive channel assignment based on local information in wireless mesh networks", Computer Engineering and Science, vol. 32, no. 8, pp. 7-10, 2010.

[7] X. C. Shi, C. H. Huang, and N. Li, "A Multiple channel assignment algorithm and routing protocol based on wireless mesh networks", Journal of Wuhan University (Natural Science Edition), vol. 57, no. 2, pp. 155-164, 2011.

[8] K. Zhang, W. Zhang, and W. Li, "Research of applicability for UAV Ad Hoc networks pre-active routing protocols", Computer Engineering and Applications, vol. 46, no. 2, pp. 4-5, 2010.

[9] J. Xu, Research on Channel Allocation and Router Technology of Mmulti-interface Wireless Network. Wu Han: Doctoral dissertation of Huazhong University of Science and Technology, 2011.

Received: September 07, 2014

Revised: October 22, 2014

Accepted: November 16, 2014

(C) Wang and Baker; Licensee Bentham Open.

This is an open access article licensed under the terms of the Creative Commons Attribution Non-Commercial License (http://creativecommons.org/licenses/by-nc/3.0/) which permits unrestricted, non-commercial use, distribution and reproduction in any medium, provided the work is properly cited. 